\title{
Potential of lacto- $N$-biose I as prebiotic for infant health: A review
}

\author{
Warsono El Kiyat ${ }^{\mathrm{a}, *}$, Santi Dwi Astuti ${ }^{\mathrm{b}}$, Slamet Budijanto ${ }^{\mathrm{c}}$, Elvira Syamsir \\ ${ }^{a}$ Department of Food Science, United Arab Emirates University, Abu Dhabi, United Arab Emirates \\ ${ }^{b}$ Department of Agricultural Technology, Jenderal Soedirman University, Purwokerto, Indonesia \\ ${ }^{c}$ Department of Food Science and Technology, IPB University, Bogor, Indonesia
}

Article history:

Received: 25 January 2021 / Received in revised form: 23 May 2021 / Accepted: 28 May 2021

\begin{abstract}
Prebiotic is one alternative in the prevention of disease in infants. Generally, it is available as oligosaccharide which may occur naturally, but can also be added as a dietary supplement for food, beverage or formula. Lacto- $N$-biose I (LNB), a kind of prebiotic has not been widely examined in regard to its activities as a bifidogenic factor. Naturally, it is available in a compound form in human milk oligosaccharide (HMO) as the main constituent of human milk rather than fat and protein. HMOs also have prebiotic activity in the body and play an important role in providing nutrition for the infant health. LNB is potential to be used in food ingredient, especially infant food formula regarding the prebiotic effect and it could be enzymatically synthesized using enzymes involved in the LNB biosynthesis pathway by microorganisms.
\end{abstract}

Keywords: Human milk oligosaccharide; infants health; lacto- $N$-biose I; prebiotic; synthesis

\section{Introduction}

Infant disease is a problem related to the nutritional status of an infant in Indonesia. Gastroenteritis is a common disease occurred in infant [1]. It can be prevented by breastfeeding that can provide functional prebiotics [2]. Prebiotics are food ingredients that can not be digested in the intestine to give benefits to the body through the development of intestinal microorganisms providing a health effect for the body. They generally improve the population of beneficial microorganisms and reduce any harmful microorganisms in the body. In a proper dosage, the consumption of prebiotics can treat and support the control of diseases such as cancer, colon cancer, constipation, diabetes mellitus, and liver. In addition, prebiotics can regulate the growth of intestinal microorganisms, prevent gastrointestinal infections, modulates the immune system, increase the bioavailability of minerals, regulate metabolic disorders associated with obesity and diabetes, and reduce the risk of cancer [3].

Human milk oligosaccharide (HMO) is a major component contained in milk. It is known to have a prebiotic activity for the growth of infant intestinal microorganisms. It is composed of oligosaccharide type 1 containing lacto- $N$-biose I (LNB) as the core structure and available in large numbers. LNB is the prebiotic candidate that has not been widely examined in regard to its activities as a bifidogenic factor. Several studies have reported that LNB significantly enhances the activity of microflora that lives in an infant's intestine [4].

Naturally, LNB exists in HMO, but the recent study revealed that it can be synthesized by enzymatic reaction [5]

* Corresponding author. Tel.: +62-877-1105-2329.

Email: warsono.el.kiyat@gmail.com

https://doi.org/10.21924/cst.6.1.2021.277 and has the potential to be used as an ingredient [6]. LNB promotes the growth and activity of intestinal microorganisms, especially in the infant's intestine.

\section{Human Milk Oligosaccharide (HMO) and Its Prebiotic Activity}

HMO is a building block of human milk in addition to lactose and fat. Its structure is very complex. HMO biosynthesis in the mammary gland starts with both the lactose core production of galactose and $\beta$-galactotransferase in the presence of $\alpha$-lactalbumin, which catalyzes glucose [7].

HMO has a varied structure and diverse bioactive properties. It consists of a combination of 5 monosaccharides constituent groups, i.e. D-glucose, $\mathrm{N}$-acetylglucosamine, Lfucose, and sialic acid. Certain monosaccharides have also been detected in other mammalian milk [8]. HMO cannot be hydrolyzed by the enzyme in the stomach and small intestine. Due to the complexity of its structure, the composition of HMO in human milk cannot be replicated in infant formula. HMO is more than just a substrate that stimulates the growth of microorganisms that are desirable in the infant's intestine [9].

\subsection{HMO affecting intestinal functions and immune protection}

Some undigested parts of HMO will pass the lower digestive tract [10], which are selectively used for the metabolism of beneficial intestinal microorganisms in an infant's body [11]. The bifidogenic activity of HMO can promote the growth of microorganisms on infant's intestine better than the formula milk [12]. HMO has some beneficial effects on the development of the neonatal intestine, such as a) promoting the growth of beneficial microorganisms; b) protection against 
infection; c) helping the development of intestinal immaturity; and d) helping intestinal adaptation to environmental conditions [13].

HMO is an inborn immune system component of human milk which protects children against infections. The protective effect of breastfeeding is caused by fucosylated of $\alpha(1-2)$ oligosaccharide bond. Fucosylated oligosaccharides can prevent diarrheal diseases through various mechanisms such as inhibiting activity of Escherichia coli toxin by binding and restricting the exposure to receptors, Campylobacter inhibition of intestinal cell adhesions and Norovirus suppression competing with intestinal epithelial tissues HMO can reduce pathogen infection with antimicrobial activity as an antiadhesive [14].

\subsection{HMO as a nutrient for brain development}

Premature infants who are given by breastfeeding have a good development at the age of 1.8 years old and a higher level of intelligence at 7 years old [15]. Brain development and cognitive are partly dependent on gangliosides containing sialic acid and polysialic acid-glycoprotein [16]. The concentration of sialic acid in the brain twice as many among the few months before birth and a few years after birth [17]. This substance is a vital ingredient for acid formation in the brain before and after the births. Human milk is rich in sialic acid and a post-mortem analysis at this stage of neonatal shows that the ganglioside and the concentration of sialic acid bound protein are significantly higher in the brain of breastfed infants than the one by formula milk fed containing low sialic acid [16]. HMO is the main carrier of sialic acid that can trigger the brain development, which plays an important role at the intelligence level in infants fed by human milk [8].

\subsection{HMO and systemic effect}

HMO is known to have systemic impacts on endothelial cell leucocyte adhesion or interaction between platelets and neutrophils. T-cells in the blood are exposed by HMO. It makes the number of IFN $\gamma$ - that produces $\mathrm{CD} 3+\mathrm{CD} 4+, \mathrm{CD} 3+\mathrm{CD} 8+\mathrm{T}$ cells increases along with Interleukin 13 coding genes that produces $\mathrm{CD} 3+\mathrm{CD} 8+\mathrm{T}$ cells. HMO affects the maturation of lymphocytes and promotes a shift in the response of T-cells to achieve the more balanced production of Th1/Th2-cytokines [7].



Fig. 1. Lacto- $N$-biose I
At the neutral fraction of HMO, lacto- $N$-fucopenatose (LNFP) III, and lacto- $N$-neotetraose (LNnT) have been shown to affect the ability of peritoneal macrophages to suppress the T-cell response of naive CD4+ [18]. LNFP III prompts the task of macrophages in vitro and increases the production of prostaglandin E2, IL-10 and TNF $\alpha$ [18]. Fucosylated HMO influences leucocyte infiltration and model flow activation using TNF $\alpha$-activated endothelial cells and leucocytes in the isolated human umbilical vein. In the ex vivo models along the fresh human blood, the formation of the neutrophil complex and the activation of neutrophils in the presence of HMO are reduced [8].

\subsection{HMO and intestinal microorganisms}

The equilibrium of microorganisms in human's intestines is one of the important factors in increasing the body's immune system [19]. Intestines are the organs that are sensitive to environmental influences. Intestinal microorganisms and the intestinal epithelium have a very close relationship, especially in the digestive system [20]. Its mechanism occurs through the walls of the intestinal mucosa and microorganisms respond both beneficial and pathogenic [7]. Therefore, it is important to note the role of HMO and its influence in the selective microorganisms growth in the intestine.

Bifidobacterium longum subsp. infantis uses HMO is the only source of carbon, whereas HMO cannot be taken up by other gut microbes. Genome Bifidobacterium longum subsp. Infantis strain ATCC 15697 comprises a substantial gene cluster composing of numerous glycosides and allegedly HMO metabolism-specific transporters [21]. The prevalence of clusters in HMO, as shown in the comparative study of genomics, correlates with their survival. Thus, not all enzymes were used in the bifidobacterial HMO metabolism at the time [22].

There are enzymes that degrade HMO in each strain bifidobacteria. The existence of such external fucosidase enzymes and lacto- $N$-biosidase produced by bacteria cells and specific transporter for LNB [Gal- $\beta-(1 \rightarrow 3)-G l c N A c]$ and GNB $[$ Gal- $\beta-(1 \rightarrow 3)-$ GalNAc] proves that Bifidobacteria with several methods can particularly utilise HMOs [23]. B. bifidum is a microorganism that can degrade the HMO with a specific mechanism. It uses LNB to hydrolyze LNT, LNB I, and lactose. LNB was subsequently released, put in a cell by $\mathrm{ABC}$ transporters specifically for LNB and converted into $\mathrm{C}_{8} \mathrm{H}_{16} \mathrm{NO}_{9} \mathrm{P}$ in the presence of lacto- $N$-biose I phosphorylase (LNBP) [24].

\section{Lacto- $N$-Biose I}

Lacto- $N$-biose I [LNB; $\operatorname{Gal}(\beta 1-3)$ GlcNAc] is an important role of disaccharide in the carbohydrate structure on several glycoconjugates (figure 1). This compound presents in HMO but it is not available in the form of a free disaccharide and active as building blocks of oligosaccharides type 1 containing lacto- $N$-tetraose as its core structure. Oligosaccharide type I is only contained in milk, and animals such as monkeys, while oligosaccharides Type II is detected in mammalian milk. In addition, LNB glycan is found in the domain of glycolipid and glycoprotein on several cell types, including epithelial cells in 
intestine and blood group antigen [25].

\subsection{Biosynthesis of LNB in bifidobacteria}

The biosynthesis of LNB specifically is done by bifidobacteria such as B. bifidum which hydrolyzes HMO type 1 outside the cell by using sialidase, fucosidase, and lacto- $N$ biosidase. Then, LNB as the outcome enters the cell by ABCtype transporter and are transformed into Gal1P (Galactose-1phosphate) and $N$-acetylhexosamine using LNBP that is first isolated from the cell-free extract of B. bifidum DSM20082.38 [26]. In the same hydrolysis activity of B. bifidum on HMO type II, it starts from the non-reducing end through the activity of sialidase, fucosidase, $\beta$-galactosidase and $\beta-N$ acetylhexosaminidase [27].

Biosynthesis of LNB in B. longum subsp. infantis directly incorporates LNT into the cytoplasm and is subsequently hydrolyzed to LNB and lactose. However, the metabolic pathway of LNB is encoded by $\ln p A B C D$ operon that encodes LNBP, NahK, GalT and GalE correspondingly [28]. On that path, LNBP phosphorylates and hydrolyzes LNB into Gal1P, are then transformed into Glu1P through GalT and GalE activity and some $\mathrm{N}$-acetylglucosamine (GlcNAc), phosphorylated via NahK produces GalNAc1P. B. bifidum has an identical operon containing two previously identified sugar kinases between the LNBP and NahK coding regions [29].

In majority of organisms galactose is metabolized to Gal1P by means of a Leloir pathway [30] consisting of four enzymes like GalM (galactokinase mutarotase) converting $\alpha$-Gal into $\beta$ Gal; GalK (galactokinase) converting $\beta$-D-Gal to Gal1P; GalT transforming Gal1P into UDP-Gal, which converts simultaneously UDP-Gal to UDP-Glc and GalE transforms UDP-Gal to UDP-Glc for the purpose of producing UDP-Glc used for re-production of GaIT. Although the GNB/LNB path is similar to the Leloir pathway, it is considered that the two pathways are different. In the Leloir pathway, for instance, $\alpha$-Gal, phosphorylated by GalK utilizes one ATP molecule, but in the LNB/GNB system, LNBP creates Gal1P without utilizing ATP through galactoside phosphorylation. It is concluded that the other sections of the LNB/GNB in its pathway differ from those of Leloir in the use of $\mathrm{N}$ acetylhexosamine. By utilizing ATP, GalNAc has been converted into GlcNAc1P. In Leloir it appears like GalK. No clusters genes in the Bifidobacterium longum NCC2705 encoding any kinds of enzyme connected with the pathway of Leloir. GalK (BL1210) and GalT (BL1211), which are discovered in the genome, except the $\ln p A B C D$ cluster, are the only gene cluster containing 2 Enzymes from Leloir's pathway. Nonetheless, Gal M, GalK, GalT and GalE are generally present in operons of other species in the genes coding enzymes of the Leloir pathway for galactose metabolism. A metabolic pathway for galactose as a carbone source for Bifidobacterium longum is used in the simplified LNB/GNB pathway. Therefore, LNB is a bifidogenic factor for intestinal tract through HMO and carbohydrate metabolism mucin, because intestinal bifidobacteria is useful for health. In addition, LNB/GNB pathway is associated with symbiosis between humans and bifidobacteria [31].

\subsection{The prebiotic activity of $L N B$}

As a Bifidus factor, the prebiotic activity of LNB is found by bifidobacteria, especially $B$. bifidum, $B$. breve and $B$. longum. However, LNB shows no effect of prebiotics on lactic acid and other bacteria (Bacteroides, Clostridium, Enterococcus, Eubacterium, Lactobacillus, Lactococcus, and Ruminococcus) [4]. In addition, LNB can be utilized by many strains of bifidobacteria comprising of 208 strains of 10 species and 4 subspecies [32]. The whole strains that naturally live in infant's gastrointestinal tract can grow by using a single LNB as a carbohydrate source, while adults, human teeth, and courageous domestic animals cannot breakdown the LNB by a strain present in the digestive tract.

As shown in table 1 , the recent study evaluated the bifidogenetic activity of lacto- $N$-biose I on the growth of microorganisms isolated from infant feces. In addition, comparative testing with other prebiotic compounds such as lactulose, raffinose, galacto-oligosaccharides, and mannanooligosaccharides was performed in vitro. The results of formula milk inoculated into the medium containing $1 \%$ LNB showed a significant improvement of the population of bifidobacteria as similar with other prebiotic compounds. But the populations of B. bifidum treated LNB $1 \%$ higher compared to the others. It is also proven from the lactic acid concentration of lower and higher acetic acid showing the larger prebiotic activity of LNB [6].

\subsection{The effect of $L N B$ on the immune system}

Based on the previous research, the LNB immunomodulatory behavior employing mice's naive immune cell expressing Receptor T-cells (TCR) especially chicken ovalbumin (OVA) demonstrated that the LNB exposure greatly decreased the Ag-specific production of IL-4 [33]. IL-4 is the leading development of the ag-specific Th2 cell in association with cytokine. LNB has the same lactose affinity according to another study[34]. Gal-3 that binds lactose can inhibit the relationship between TCR and Gal-3. It changes the effect of Gal-3, which binds LNB to damage the function partially and only reduce the secretion of Ag-specific IL-4. In general, by the modification of APCs signal, LNB affects Ag-specific immune response [33].

\section{Synthesis of Lacto- $N$-Biose I}

LNB synthesis was first introduced by Flowers and Jeanloz [35] that produced the derivatives of muramic acid and $\mathrm{N}$ acetylmuramic acid. LNB synthesis was carried out to produce the products with high yields and high purity. This was due to the result of LNB synthesis used as a food ingredient that may provide a prebiotic effect on the formula. In addition, it took a shorter time to produce LNB for increasing productivity. Figure 2 represents a comparison between biosynthetic pathway in human and enzymatic synthesis of lacto- $N$-biose I [36-37].

\subsection{Enzymatic synthesis of $L N B$}

Research related to synthesis of lacto- $N$-biose I was in developed [5,26,28,36-37]. As briefly seen in table 2 . LNB can 
be synthesized using glycosynthase isolated from Thermus thermophilus through a trans-glycolytic mechanism [5]. The synthesis of LNB in scale-up has been conducted. It produced LNB up to $1.4 \mathrm{~kg}$ of sucrose and GlcNAc with the addition of UDP-Glc and phosphates using 4 enzymes such as sucrose phosphorylase (SP), GalT, GalE and LNBP as the catalyst for 700 hours [28]. Such methods could be expected to be used on an industrial scale using a bioreactor system with an immobilized enzyme. In addition, the present invention is applicable to strengthen the hypothesis that in infant formulas, LNB may be employed as food additives similar to the LNB content in human milk.

LNB synthesis according to Nishimoto and Kitaoka started with the phosphorylation of sucrose into $\alpha$-glucose-1phosphate $(\mathrm{Glc} 1 P)$ with the addition of SP as a catalyst. Glc1P was transformed into UDP-Glc at the same time that UDP-Gal is transformed into Gal1P by GalT. As a result of the GalE behavior, the UDP-Glc was changed into UDP-Gal, where GalT was utilized by the UDP-Gal. LNB and then synthesized from Gal1P and GlcNAc by LNBP [28].

\subsection{The enzyme involved in LNB synthesis}

The enzyme acts as a catalyst in a reaction. In certain conditions, the chemical reactions that run in the presence of a catalyst can be done faster. In LNB synthesis, enzyme plays an important role in catalyzing the chemical reactions. Enzymes that contribute to LNB synthesis so far quite a lot, dependent on the synthesis method. Some enzymes are generally present in the LNB pathway occurred in the infant's intestinal microorganisms to degrade LNB as a carbon source to stimulate activity and growth. Lacto- $N$-Biose I Phosphorylase (LNBP; $1,3-\beta$-galactosyl- $N$-acetylhexosamine phosphoryl-ase) is active to phosphorylate LNB into Gall $P$ in the presence of GlcNAc. In CAZy database, LNBP is classified into glycoside hydrolase family 112 based on similarity of structure with the $\beta$-galactosidase GH42. Phosphorylase generally can hydrolyze sugar as the substrate which plays an important role as a source of energy, particularly for anaerobic bacteria. This is because phosphorylation directly produces sugar phosphorylated without requiring ATP. This energy can be obtained from the substrate which has greater energy than other sugars, especially in an anaerobic condition that only has 3 ATP molecules available through a glycolytic pathway from glucose 6phosphate [24].

LnpB has shown an interaction in the production of using GlcNAc and ATP, $\alpha$-GlcNAc 1-phosphate (GlcNAc1P) and it indicated that $\operatorname{LnpB}$ was a novel kinase anomeric $(\mathrm{NahK}$; $N$-acetylhexosamine 1-kinase) [31]. This protein showed a similar action on GlcNAc which produces GlcNAc1P with low activity in several monosaccharides. NahK had a number EC2.7.1.162 and was active in hexose, which phosphorylated its substrate in position 6 .

$\operatorname{LnpC}$ and $\ln p D$ have been demonstrated as GalT (UDPglucose-hexose 1-phosphate-uridylyltransferase; EC 2.7.7.12) and GalE (UDP-glucose 4-epimerase; EC 5.1.3.2) amino acid

Table 1. The prebiotic effect of LNB in several experiments

\begin{tabular}{|c|c|c|}
\hline Microorganism & Information & Results \\
\hline $\begin{array}{l}\text { Bifidobacterium longum subsp. infantis } \\
\text { ATCC15697 [40] }\end{array}$ & $\begin{array}{l}\text { Comparison of the substrate specificity of LNB } \\
\text { with pNP- } \beta \text {-Gal, pNP- } \beta \text {-Fuc, lactose, LacNAc, } \\
\text { LNnT, and LNT }\end{array}$ & $\begin{array}{c}k_{c a t} / \mathrm{K}_{\mathrm{M}} \text { value of LNB was higher, but it was lower } \\
\text { than LNT, lactose, and LacNAc }\end{array}$ \\
\hline $\begin{array}{l}208 \text { strains consisted of } 10 \text { species and } 4 \\
\text { subspecies of Bifidobacteria [32] }\end{array}$ & $\begin{array}{l}\text { LNB used as a source of carbon addition of } \ln p A \\
\text { gene (LNBP) }\end{array}$ & $\begin{array}{c}\text { Strains of Bifidobacteria can growth. } B \text {. } \\
\text { pseudocatenulatum, B. animalis subsp. animalis, } \\
\text { B. pseudolongum can utilize LNB }\end{array}$ \\
\hline $\begin{array}{l}18 \text { strains of Bifidobacteria, Clostridium, } 13 \\
\text { Enterobacter and } 8 \text { lactic acid bacteria [4] }\end{array}$ & $\begin{array}{l}\text { Bacteria were inoculated into media containing } \\
\mathrm{LNB} \text { and the absorbance was evaluated in } \mathrm{OD}_{60}\end{array}$ & $\begin{array}{l}\text { B. bifidum JCM1254 and JCM7004, B longum } \\
\text { subsp. infantis JCM1210 and JCM1222 }{ }^{\mathrm{T}}, \text { B. } \\
\text { longum subsp. longum } \mathrm{JCM} 1217^{\mathrm{T}} \text { and } \\
\text { JACM7054, B. breve JCM1192 }{ }^{\mathrm{T}} \text { and } B \text {. scardovii } \\
\text { JCM12489 }{ }^{\mathrm{T}} \text { growth } 2 \mathrm{x}>\text { control }\end{array}$ \\
\hline Bifidobacteria [6] & $\begin{array}{l}\text { Comparing the prebiotic effect of LNB with other } \\
\text { prebiotics: lactulose, raffinose, galacto- } \\
\text { oligosaccharide, and mannano-oligosaccharide }\end{array}$ & $\begin{array}{l}\text { The population of Bifidobacteria given LNB } \\
\text { significantly grow as large as other prebiotics. } \\
\text { The activity of } B \text {. bifidum was higher than other } \\
\text { prebiotics. The residual compound of LNB } \\
\text { treatment such as lactic acid was lower while } \\
\text { acetic acid was higher than other prebiotics }\end{array}$ \\
\hline Lactobacillus casei [41] & $\begin{array}{l}\text { The activity of gen cluster Gnb REFGBCDA was } \\
\text { evaluated on several substrates, including LNB }\end{array}$ & $\begin{array}{l}\text { LNB was hydrolyzed on Gnb G (phospho- } \beta \text { - } \\
\text { galactosidase from L. casei) }\end{array}$ \\
\hline
\end{tabular}



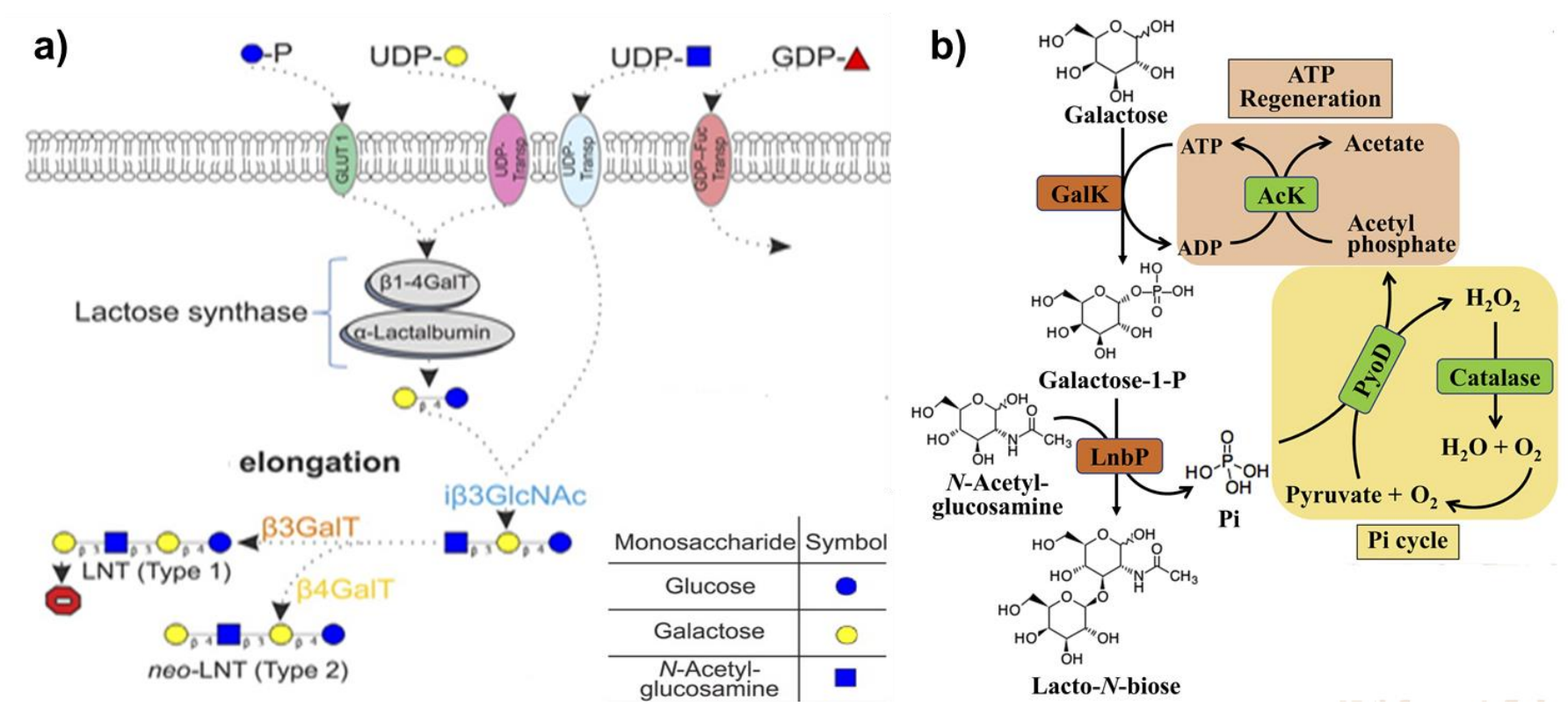

Fig. 2. Comparison of biosynthesis pathway (a) and enzymatic synthesis of lacto- $N$-biose I (b). LNB is connected to the the galactosyl moiety of terminal lactose through a $\beta$-1,3-binding with an extra $\beta$-1,6-binding in branched HMO.

Table 2. Enzymatic synthesis of LNB in several experiments

\begin{tabular}{|c|c|c|c|}
\hline Microorganism Source & Enzyme & Substrate & Yield (\%) \\
\hline Xanthomonas manihotis [38] & $\beta$-D-galactosidase & $\begin{array}{l}\text { p-nitophenyl } \beta \text {-D-galactopyranoside dan 2- } \\
\text { acetoamido-2-deoxy-D-glucopyranose }\end{array}$ & 22 \\
\hline Bifidobacterium bifidum [26] & Lacto- $N$-biose I (LNBP) & Gal1P and GlcNAc & 76 \\
\hline Xanthomonas manihotis [39] & $\beta$-D-galactosidase & $\begin{array}{l}p \text {-nitophenyl } \beta \text {-D-galactopyranoside dan 2- } \\
\text { acetoamido-2-deoxy-D-glucopyranose }\end{array}$ & 55 \\
\hline Bifidobacterium bifidum [31] & $\begin{array}{l}\text { Sucrose phosphorylase, GalT, GalE, and } \\
\text { LNBP }\end{array}$ & GlcNAc and Gal1P & 96.9 \\
\hline Thermus thermophilus [5] & Transglycosidase & GlcNAc & 80 \\
\hline $\begin{array}{l}\text { Bifidobacterium longum and Ruminoccus } \\
\text { albus NE1 [42] }\end{array}$ & LNBP and lactose phosphorylase & GlcNAC and Lactose & 75.3 \\
\hline
\end{tabular}

sequence based accordingly. GalT and GalE are galactose metabolic enzymes associated in the Leloir pathway. Both of them become active in the change of Gal1P into Glc1P and allow the substrate to enter into the glycolytic pathway. Gal1P can be formed from LNB through phosphorylation by LNBP with GalT and GalE, indicating the transformation into Glc1P of LNB galactose component provided by an enzyme expressed in the gene cluster that produced energy [24].

Sucrose phosphorylase (SP) plays an important role in LNB synthesis on Nishimoto and Kitaoka's method, which is active as a catalyst which phosphorylates sucrose into UDP-Glc. In this method, SP with the pET30 vector can process LNB synthesis from sucrose with the addition of UDP-Glc GlcNAc and phosphates to produce a yield of $99.6 \%$. This enzyme has a number EC 2.4.1.7 [31].

Transglycosidase (1,4- $\alpha$-D-glucan-(D-glucose)6- $\alpha-\mathrm{D}$ glucosyltransferase) can be used for LNB synthesis. This enzyme obtained from Thermus thermophilus that catalyzes transglycosylation of phenyl-2-amino-1-thio- $\beta$-Dglucopyranoside as the substrate into phenyl $\beta$-Dgalactopyranosyl- (1-3)-2- deoxy -2-amino-1-thio- $\beta$-Dglucopyranoside which produces 2 -acetamido analog and tipped LNB formation as the final product [5]. This enzyme has a number EC 2.4.1.24. 


\section{Conclusion}

$\mathrm{HMO}$ is one of the major components of milk playing an important role as a source of nutrients for the infant's health as well as prebiotics for infant intestinal microorganisms to improve health. LNB is a disaccharide that is bound to the core structure of HMO contained in large numbers. In addition, LNB has a prebiotic activity by stimulating growth and activity of microorganisms such as bifidobacteria which are useful for health. LNB has a great potential to be used as a food ingredient, especially for infant formula. In addition, LNB can be synthesized enzymatically, chemically, and chemoenzymatically using enzymes involved in the biosynthesis pathway LNB by microorganisms. The synthesis of good lacto-N-biose I has been widely used to obtain high productivity and efficiency. Although some methods require long processing times with complex stages, a continuous system or a one-pot system can be used to solve this problem. For the future prospect, LNB production on a large scale also needs to be carried out for commercialization purposes. In addition, as a prebiotic candidate, lacto-N-rose I has the prospect of being used as a food ingredient because of its functionality. This is related to its application as a food additive in infant formula. Therefore, regulations related to their use need to be issued to increase the potential for their use in food.

\section{References}

1. M. Bartick and A. Reinhold, The burden of suboptimal breastfeeding in the United States: A pediatric cost analysis, Pediatrics 125 (2010) e1048-e1056.

2. Y. Vandenplas et al., Probiotics and prebiotics in prevention and treatment of diseases in infants and children, J. Pediatr 87 (2011) 292-300

3. K. Venema and A. P. do Carmo, Probiotics and prebiotics: Current research and future trends. Norfolk: Caister Academic Press, 2015.

4. M. Kiyohara, A. Tachizawa, M. Nishimoto, M. Kitaoka, H. Ashida, and K. Yamamoto, Prebiotic effect of Lacto- $N$-biose I on Bifidobacterial Growth, Biosci. Biotechnol. Biochem. 73 (2009) 1175-1179.

5. A. D. D'Almeida, M. Ionata, V. Tran, C. Tellier, M. Dion, and C. Rabiller, An expeditious and efficient synthesis of $\beta-D$ galactopyranosyl-(1->3)-D- N-acetylglucosamine (lacto- N-biose) using a glycosynthase from Thermus thermophilus as a catalyst, Tetrahedron: Asymmetry 20 (2009 1243-1246.

6. T. Satoh, T. Odamaki, M. Namura, T. Shimizu, and K. Iwatsuki, In vitro comparative evaluation of the impact of lacto-N-biose I, a major building block of human milk oligosaccharides, on the fecal microbiota of infants, Anaerobe 19 (2013) 50-57.

7. C. Kunz, S. Kuntz, and S. Rudloffl, Bioactivity of human milk oligosaccharides, in Food Oligosaccharides: Production, Analysis, and Bioactivity, 1st ed., J. Moreno and M. Sanz, Eds. New York: JohnWiley and Sons, Ltd., 2014, pp. 5-20.

8. L. Bode, Human milk oligosaccharides: every baby needs a sugar mama, Glycobiology 22 (2012) 1147-1162.

9. D. L. Oliveira, R. A. Wilbey, A. S. Grandison, and L. B. Roseiro, Milk oligosaccharides: A review, Int. J. Dairy Technol. 68 (2015) 305-321.

10. P. Chaturvedi, C. Warren, C. Buescher, L. Pickering, and D. Newburg, Survival of human milk oligosaccharides in the intestine of infants, in Bioactive Components of Human Milk. Advances in Experimental
Medicine and Biology, Vol 501, D. Newburg, Ed. Boston: Springer, 2001, pp. 315-323.

11. R. E. Ward, M. Nin, D. A. Mills, C. B. Lebrilla, and J. B. German, In vitro fermentation o $f$ breast milk oligosaccharides by Bifidobacterium infantis and Lactobacillus gasseri, Appl. Environ. Microbiol. 72 (2006) 4497-4499.

12. H. Harmsen et al., Analysis of intestinal flora development in breastfed and formula-fed infants by using molecular identification and detection methods, J. Pediatr. Gastroenterol. Nutr. 30 (2000) 61-67.

13. S. M. Donovan, Human milk components in gastrointestinal development: Current knowledge and future needs, J. Pediatr. 149 (2006) S49-S61.

14. D. S. Newburg, G. M. Ruiz-Palacios, and A. L. Morrow, Human milk glycans protect infants against enteric pathogens, Annu. Rev. Nutr. 25 (2005) 37-58.

15. A. Lucas, R. Morley, T. Cole, G. Lister, and C. Leeson-Payne, Breast milk and subsequent intelligence quotient in children born preterm, Lancet, 339 (1992) P261-P264.

16. B. Wang, Sialic acid is an essential nutrient for brain development and cognition sialic acid is an essential nutrient for brain development and cognition, Annu. Rev. Nutr. 29 (2009) 177-222.

17. L. Svennerholm, K. Bostrom, P. Fredman, J. Mansson, B. Rosengren, and B. Rynmark, Human brain gangliosides: developmental changes from early fetal stage to advanced age, Biochim. Biophys. Acta 1005 (1989) 109-117.

18. O. Atochina and D. Harn, LNFPIII/LeX-stimulated macrophages activate natural killer cells via CD40-CD40L interaction, Clin. Diagn. Lab. Immunol. 12 (2005) 1041-1049.

19. A. W. Walker et al., Dominant and diet-responsive groups of Bacteria within the human colonic microbiota, ISME J. 5 (2010) 220-230.

20. C. L. Maynard, C. O. Elson, R. D. Hatton, and C. T. Weaver, Reciprocal interactions of the intestinal microbiota and immune system, Nature 489 (2012) 231-241.

21. A. Marcobal et a A. Marcobal et al., Bacteroides in the infant gut consume milk, Cell Host Microbe 10 (2011) 507-514.

22. G. Hybridization, R. G. Locascio, P. Desai, D. A. Sela, B. Weimer, and D. A. Mills, Broad conservation of milk utilization genes in Bifidobacterium longum subsp. infantis as revealed by comparative, Appl. Environ. Microbiol. 76 (2010) 7373-7381.

23. S. Asakuma et al., Physiology of consumption of human milk oligosaccharides by infant gut-associated Bifidobacteria, J. Biol. Chem. 286 (2011) 34583-34592.

24. M. Kitaoka, Bifidobacterial enzymes involved in the metabolism of human milk oligosaccharides, Adv. Nutr. 3 (2012) 422S-429S.

25. S. Hakomori, Structure and function of glycosphingolipids and sphingolipids: Recollections and future trends, Biochim. Biophys. Acta 1780 (2008) 325-346.

26. D. Derensy-Dron, F. Krzewinski, C. Brassart, and S. Biologique, $\beta-1,3-$ galactosyl-N-acetylhexosamine phosphorylase from Bifidobacterium bifidum DSM 20082: Characterization, partial purification, and relation to mucin degradation, Biotechnol. Appl. Biochem. 10 (1999) 3-10.

27. M. Miwa and T. Horimoto, Cooperation of $\beta$-galactosidase and $\beta$ - $N$ acetylhexosaminidase from Bifidobacteria in Assimilation of Human Milk Oligosaccharides with Type 2 Structure, Glycobiology 20 (2010) 1402-1409.

28. M. Nishimoto and M. Kitaoka, Identification of N-Acetylhexosamine 1kinase in the complete lacto-N-biose I/galacto-N-biose betabolic pathway in Bifidobacterium longum, Appl. Environ. Microbiol. 73 (2007) 6444-6449.

29. F. De Bruyn, J. Beauprez, J. Maertens, W. Soetaert, and M. De Mey, Unraveling the Leloir pathway of Bifidobacterium bifidum: 
significance of the uridylyltransferases, Appl. Environ. Microbiol. 79 (2013) 7028-2035

30. K. Fujita et al., Identification and molecular cloning of a novel glycoside hydrolase family of core 1 type $O$-glycan-specific endo- $\alpha$ $N$-acetylgalactosaminidase from Bifidobacterium longum, J. Biol. Chem. 280 (2005) 37415-37422.

31. M. Nishimoto and M. Kitaoka, Practical preparation of lacto- $\mathrm{N}$-biose $\mathrm{I}$ , a candidate for the bifidus factor in human milk, Biosci. Biotechnol. Biochem. 71 (2007) 2101-2104.

32. J. Xiao et al., Distribution ofin vitro fermentation ability of lacto- $N$ biose $i$, a major building block of human milk oligosaccharides, in Bifidobacterial strains, Appl. Environ. Microbiol. 76 (2010) 54- 59.

33. M. Goto, Y. Takano-ishikawa, M. Nishimoto, and M. Kitaoka, Effect of lacto-N-biose $I$ on the antigen-specific immune responses of splenocytes, Biosci. Microbiota, Food, Heal. 31 (2012) 47-50.

34. S. Fort, H. Kim, and O. Hindsgaul, Screening for galectin-3 inhibitors from synthetic lacto- $N$-biose libraries using microscale affinity chromatography coupled to mass spectrometry, J. Org. Chem. 71 (2006) 7146-7154.

35. H. M. Flowers and R. W. Jeanloz, The aynthesis of 2-acetamido-2deoxy-3-O-( $\beta$-D-galactopyranosyl $)-\alpha-D$-glucose, J. Org. Chem. 28 (1963) 1377-1379.

36. D. Blank, V. Dotz, R. Geyer, and C. Kunz, Human milk oligosaccharides and Lewis blood group: Individual high-throughput sample profiling to enhance conclusions from functional studies,
Adv. Nutr.
3 (2012) 440S-449S

37. Z. Du et al., Lacto-N-biose synthesis via a modular enzymatic cascade with ATP regeneration, Science 24 (2021) 102236.

38. H. Fujimoto, Regioselective Synthesis of $\beta$-D-Gal-from Xanthomonas manihotis, J. Carbohydr. Chem., vol. 16, no. 6, pp. 37-41, 2006.

39. A. Vetere, M. Miletich, M. Bosco, and S. Paoletti, Regiospecific glycosidase-assisted synthesis of lacto-N-biose I (Galß1-3GlcNAc) and 3'-sialyl-lacto-N-biose I (NeuAca2 -3Galß1-3GlcNAc), Eur. J. Biochem. 247 (2000) 942-949.

40. E. Yoshida, H. Sakurama, and M. Kiyohara, Bifidobacterium longum subsp. infantis uses two different $\beta$-galactosidases for selectively degrading type-1 and type-2 human milk oligosaccharides, Glycobiology 22 (2012) 361-368.

41. G. N. Bidart, J. Rodríguez-díaz, V. Monedero, and M. J. Yebra, $A$ Unique Gene Cluster for the Utilization of the Mucosal and Human MilkAssociated Glycans Galacto-N-Biose and Lacto-N-Biose in Lactobacillus casei, Mol. Microbiol. 93 (2014) 521-538.

42. W. E. Kiyat, Enzymatic Synthesis of Lacto-N-Biose I from Lactose and GlcNAc using Lactose Phosphorylase and Lacto-N-Biose I Phosphorylase, Bogor Agricultural University, Indonesia, 2017. 